\title{
ACCURACY OF THE HEMATOLOGICAL SCORING SYSTEM (HSS) FOR THE DIAGNOSIS OF NEONATAL SEPSIS IN TERTIARY CARE HOSPITAL.
}

1. MBBS, FCPS (Paeds Medicine) Clinical Fellow Neonatology Quaid-I-Azam Medical College/ Civil Hospital Bahawalpur.

2. MBBS, FCPS (Pediatric Medicine) Senior Registrar Pediatric Medicine Quaid-I-Azam Medical College/ Civil Hospital Bahawalpur.

3. MBBS, FCPS (Pediatric Medicine),

FCPS (Neonatology)

Assistant Professor Neonatology Quaid-I-Azam Medical College/ Civil Hospital Bahawalpur.

Correspondence Address: Dr. Muhammad Asghar Ali Clinical Fellow Neonatology Quaid-I-Azam Medical College/ Civil Hospital Bahawalpur. gdrmasghar@yahoo.com

Article received on: 13/04/2019 Accepted for publication: $26 / 07 / 2019$

\section{Muhammad Asghar Ali ${ }^{1}$, Ali Hammad ${ }^{2}$, Hafiz Muhammad Anwar ${ }^{3}$}

ABSTRACT... Objectives: Neonatal sepsis is a known and significant contributor to newborn morbidity and mortality. We conducted this study to find out the efficiency of hematological scoring system (HSS) in predicting neonatal sepsis. Study Design: Prospective study. Setting: Neonatology Unit of Civil Hospital, Bahawalpur. Period: $1^{\text {st }}$ July 2018 to $31^{\text {st }}$ December 2018. Materials \& Methods: A total of 100 neonates having high probability of sepsis were admitted and evaluated in NICU, Institution's pathological lab was the center for all the workups. Sensitivity, specificity, positive predictive values (PPVs) along with negative predictive values (NPVs) were calculated for different study parameters. $P$ value $<0.05$ was considered as significant. Results: Amongst a total of 100 neonates, $62(62.0 \%)$ were males and $38(38.0 \%)$ females. There were $32(32.0 \%)$ preterm while $68(68.0 \%)$ term neonates. Culture positive cases were found to be $33(33.0 \%)$ whereas CRP was noted reactive in $62(62.0 \%)$ cases. Neonatal age as preterm, culture positive neonates $(p<0.001)$ as well as CRP as reactive were found to be significantly associated with HSS $>5$. Immature to total neutrophil ratio (I:T) as well as Immature to mature neutrophil ratio (I:M) had the highest sensitivity and specificity. Conclusion: HSS is a simple to use and efficient method that can be used to early diagnose and treat cases of neonatal sepsis. HSS has a high sensitivity as well specificity as high scores of HSS highlight sepsis.

Key words: $\quad$ CRP, Culture, HSS, Neonatal Sepsis.

Article Citation: Ali MA, Hammad A, Anwar HM. Accuracy of the hematological scoring system (HSS) for the diagnosis of neonatal sepsis in Tertiary Care Hospital. Professional Med J 2020; $27(4): 742-745$.

DOI: 10.29309/TPMJ/2020.27.04.3546

\section{INTRODUCTION}

Neonatal sepsis is a known and significant contributor to newborn morbidity and mortality. The problem resonates more when we consider the impact of neonatal sepsis in developing countries. The prevalence of neonatal sepsis is around $12 \%$ with $16 \%$ mortality amongst all neonatal deaths. ${ }^{1}$ Neonatal sepsis affects neonates more as compared to children of other age groups. Infections are the main cause of neonatal sepsis including both early or late onset. Most cases of early onset sepsis are observed within the $1^{\text {st }}$ day of life, and significantly low proportion afterwards. ${ }^{2}$

Trasplacental route or infections from passage through birth canal or infected blood are some of the most common possibilities for neonatal sepsis. Weak immune system of newborns make them more vulnerable to infections as compared to other age groups. ${ }^{3}$ Many a times, sepsis is accompanied with non-specific features which makes its early diagnosis difficult, thus delaying rationale management. ${ }^{4}$ Blood culture is considered to be the gold standard for the diagnosis but it takes around 2 to 3 days and not always available. Hematological scoring system (HSS) of Rodwell and colleagues ${ }^{5}$, consisting of hematological parameters are cheap, easy use and non-complex. Over the years, HSS has shown to be an effective for prediction and recognition of neonatal sepsis. Not much work in Pakistan has been done to evaluate the efficiency of HSS for diagnosis of neonatal sepsis so we wanted to conduct this study to find out the efficiency of HSS in predicting neonatal sepsis.

\section{MATERIAL AND METHODS}

A total of 100 neonates with high probability of neonatal sepsis admitted to Neonatology unit of 
Civil Hospital, Bahawalpur were considered for this prospective study from $1^{\text {st }}$ July 2018 to $31^{\text {st }}$ December 2018.

Institution's pathological lab was the center for all the workups. Having aseptic conditions, 0.5-1 $\mathrm{ml}$ of blood sample was taken through peripheral venipuncture. Complete blood counts, culture and hematological scores were done and peripheral smears were evaluated. Scoring was done using HSS of Rodwell and colleagues. ${ }^{5}$ One score is allotted for every one of seven findings. Abnormal total leukocyte count, abnormal total polymorphonuclear neutrophis (PMN) count, raised immature $\mathrm{PMN}$ count, raised immature to total (I:T) PMN ratio, immature to mature (I:M) PMN ratio $\geq 0.3$, platelet count as less than or equal to150,000/cumm and amplified degenerative or toxic changes in PMNs were recorded. An abnormal total PMN count was given a score of 2 instead of 1 if no mature polymorphs were observed on the peripheral smear to compensate for the low I:M ratio. Immature polymorphs comprised promyelocyte, myelocyte, metamyelocytes, and band forms. Band cell were labelled as a PMN where nucleus was indented by more than one-half, but isthmus in lobes was wide enough to disclose 2 distinct margins with nuclear content in it. Degenerative changes contained vacuolization, toxic granulations and Dohle bodies. Score of $\leq 2$ is considered as lower risk; score 3-4 as moderate risk; and score $\geq 5$ as higher risk for developing sepsis.

Sensitivity, specificity, PPVs and NPVs were calculated for each parameter. $\mathrm{P}$ value was also calculated for different parameters. Permission from institute's ethical committee was sought before the commencement of this study and informed consent was attained from parents/ guardians of all neonates.

\section{RESULTS}

Amongst a total of 100 neonates, 62 (62.0\%) were males and $38(38.0 \%)$ females. There were 32 (32.0\%) preterm while 68 (68.0\%) term neonates. Culture positive cases were found to be 33 (33.0\%) whereas CRP was noted reactive in 62 $(62.0 \%)$ cases.

There was no difference amongst cases when HSS was compared with regards to gender ( $p$ value $>0.380$ ). Neonatal age as preterm was found to be statistically significant ( $p$ value < 0.001 ) as out of 33 neonates with HSS > 5, 29 (87.9\%) were preterm. All 33 neonates with HSS $>5$ were turned out to be culture positive ( $p$ $<0.001)$. CRP was found to be reactive in 27 (81.8\%) neonates out of total of 33 with HSS of $>5(p<0.001)$. All neonates with HSS of $<2$ had their CRP non reactive.

Different tests were evaluated for sensitivity, specificity, PPV and NPV, NPV. For TLC, NPV was highest (84.8\%) while PPV was lowest (52.9\%). For ANC, specificity was highest $(91.7 \%)$ while sensitivity was lowest (48.6\%). For I:T, NPV was highest $(93.2 \%)$ whereas specificity was lowest (90.1\%). For I:M, specificity was highest (94.8\%) while PPV was lowest (91.7\%). For Platelets, PPV was highest $(61.2 \%)$ whereas sensitivity was lowest $(52.8 \%)$.

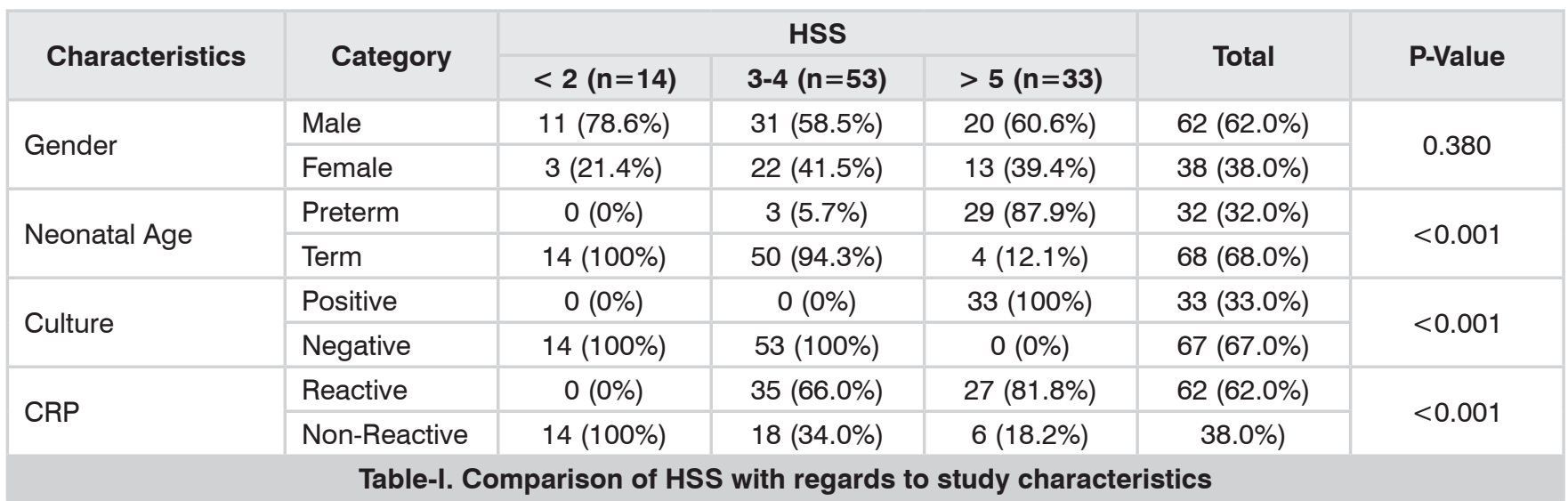




\begin{tabular}{|l|c|c|c|c|}
\hline \multicolumn{1}{|c|}{ Parameters } & Sensitivity (\%) & Specificity (\%) & PPV (\%) & NPV (\%) \\
\hline TLC & 68.2 & 72.8 & 52.9 & 84.8 \\
\hline ANC & 48.6 & 91.7 & 45.7 & 90.7 \\
\hline I:T & 92.4 & 90.1 & 92.4 & 93.2 \\
\hline I:M & 92.6 & 94.8 & 91.7 & 93.2 \\
\hline Platelet & 52.8 & 58.9 & 61.2 & 58.4 \\
\hline
\end{tabular}

\section{DISCUSSION}

Advancement regarding antibiotics as well as improved medical services has made much progress regarding successful treatment of neonatal sepsis in the last few decades but still it remains one of the leading cause of neonatal morbidity and mortality. ${ }^{6}$ Neonatal sepsis cannot be diagnosed precisely unless blood culture is performed but the process can delay the overall management. Excessive and undue use of antibiotics in the recent years have been a very debatable topic ${ }^{7}$ so a diagnostic test that has a higher sensitivity is most desired.

In the present study, neonatal age as preterm was found to be statistically significant $(p<0.001)$ as out of 33 neonates with HSS > 5, 29 (87.9\%) were preterm. Our results were found to be pretty consistent with those done earlier. ${ }^{4,8}$ preterm are believed to be more at risk for infections as compared to term neonates that can possibly be due to their weak immunity as well as lower levels of ummunoglobulins and les birth weight. ${ }^{8}$

We noted all 33 neonates with HSS $>5$ were turned out to be culture positive ( $p<0.001$ ). HSS $>5$ has been found significantly associated with confirmed sepsis previously where in an Indian setting 8 , it was noted that $83 \%$ cases of HSS $>5$ had confirmed sepsis. Bhalodia MJ and colleagues $^{6}$ also found that all cases with HSS > 5 had culture proven sepsis in their study.

In our study, raised I:T and IM ratio turned out to be the best parameters for diagnosing sepsis. Our results in this regard stand similar to what other researchers have found. ${ }^{9-11}$ Thrombocytopenia was noted to be commonly linked with sepsis but with poor prognosis. This could be because raised platelet devastation secondary to infection, collapse of platelet production mechanism because of decreased megakaryocytes or it could be related to endotoxins. ${ }^{8,12,13}$

A study from Bijapur India ${ }^{6}$ demonstrated that TLC had $75 \%$ specificity with poor sensitivity $(67 \%)$. The same study found ANC to have excellent specificity $92 \%$ but sensitivity turned out to be quite low46\%. That study proved that I:T and I:M had the highest sensitivity and specificity (>91\%) which further assert results of the current study.

The current study and few others have amplified the benefits of HSS in precisely diagnosing neonatal sepsis. It can be used as a screening tool to diagnose sepsis with a simple and time effective criteria. HSS can also be used effectively to find out non-infected cases with a high sensitivity as well specificity as high scores of HSS highlight sepsis.

\section{CONCLUSION}

HSS is a simple to use and efficient method that can be used to early diagnose and treat cases of neonatal sepsis. HSS has a high sensitivity as well specificity as high scores of HSS highlight sepsis.

\section{Copyright $\odot 26$ July, 2019.}

\section{REFERENCES}

1. Sankar MJ, Agarwal R, Deorari AK, Paul VK. Sepsis in the newborn. Indian J Pediatr 2008; 75:261-6.

2. Behrman RE, Ktregman RM, Jeanson $H$, Nelson Text Book of Pediatrics. 17th ed. Philadelphia: W.B. Saunders Publishers; 2004. p. 811-4.

3. Robertson NR. Text Book of Neonatology by Robertson. 2nd ed, Philadelphia: Churchill Livingstone Publishers; 1992. p. 925-99.

4. Bhat YR, Rao A. Sepsis screen in early neonatal sepsis. J Clin Diagn Res 2010; 4:3331-6. 
5. Rodwell RL, Leslie AL, Tudehope DI. Early diagnosis of neonatal sepsis using a hematologic scoring system. J Pediatr 1988; 112:761-7.

6. Bhalodia MJ, Hippargi SB, Patil MM. Role of hematological scoring system in diagnosis of neonatal Sepsis. J Clin Neonatol 2017; 6:144-7.

7. Narasimha A, Kumar ML. Significance of hematological scoring system (HSS) in early diagnosis of neonatal sepsis. Indian J Hematol Blood Transfus 2011; 27(1):14-7.

8. Makkar M, Gupta C, Pathak R, Garg S, Mahajan NC. Performance evaluation of hematologic scoring system in early diagnosis of neonatal sepsis. J Clin Neonatol 2013; 2:25-9.

9. Ghosh S, Mittal M, Jaganathan G. Early diagnosis of neonatal sepsis using a hematological scoring system. Indian J Med Sci 2001; 55:495-500.

10. Khair KB, Rahman MA, Sultana T, Roy CK, Rahman $M Q$, Shahidullah $M$, et al. Role of hematologic scoring system in early diagnosis of neonatal septicemia. BSMMU J 2010; 3:62-7.

11. Narasimha A, Harendra Kumar ML. Significance of hematological scoring system (HSS) in early diagnosis of neonatal sepsis. Indian J Hematol Blood Transfus 2011; 27:14-7.

12. Sriram R. Correlation of blood culture results with the sepsis score and sepsis screen in the diagnosis of neonatal sepsis. Int J Biol Med Res 2011; 2:360-8.

13. Manroe BL, Weinberg AG, Rosenfeld CR, Browne R. The neonatal blood count in health and disease. I. Reference values for neutrophilic cells. J Pediatr 1979; 95:89-98.
14. Anwer SK, Mustafa S. Rapid identification of neonatal sepsis. J Pak Med Assoc 2000; 50:94-8.

15. Bellig L, Ohning B. Neonatal Sepsis. E Medicine Journal Pediatrics/ Neonatology. 2003; 4(1).6. Thilo EH, Rosenberg AA. Infections of the Newborn. Current pediatric diagnosis and treatment, 14th edition. Hay WW, Hayward AR, Levin MJ, Sondheimer JM: editors. New Jersey: Appleton and Lange, 1999.

16. Manroe BL, Weinberg AG, Rosenfeld CR, Browne R. The neonatal blood count in health and disease. I. Reference values for neutrophilic cells. J Pediatr 1979; 95:89-98.

17. Akenzua GI, Hui YT, Milner R, Zipursky A. Neutrophil and band counts in the diagnosis of neonatal infections. Pediatrics 1974; 54:38-42.

18. Zipursky A, Palko J, Milner R, Akenzua Gl. The hematology of bacterial infections in premature infants. Pediatrics 1976; 57:839-53.

19. Speer CP, Gahr M, Schröter W. Early diagnosis of neonatal infection. Monatsschr Kinderheilkd 1985; 133:665-8.

20. Philip AG, Hewitt JR. Early diagnosis of neonatal sepsis. Pediatrics 1980; 65:1036-41.

21. Basu S, Guruprasad, Narang A, Garewal G. Diagnosis of sepsis in the high risk neonate using a hematologic scoring system. Indian J Hematol Blood Transf 1999; 17:32-4.

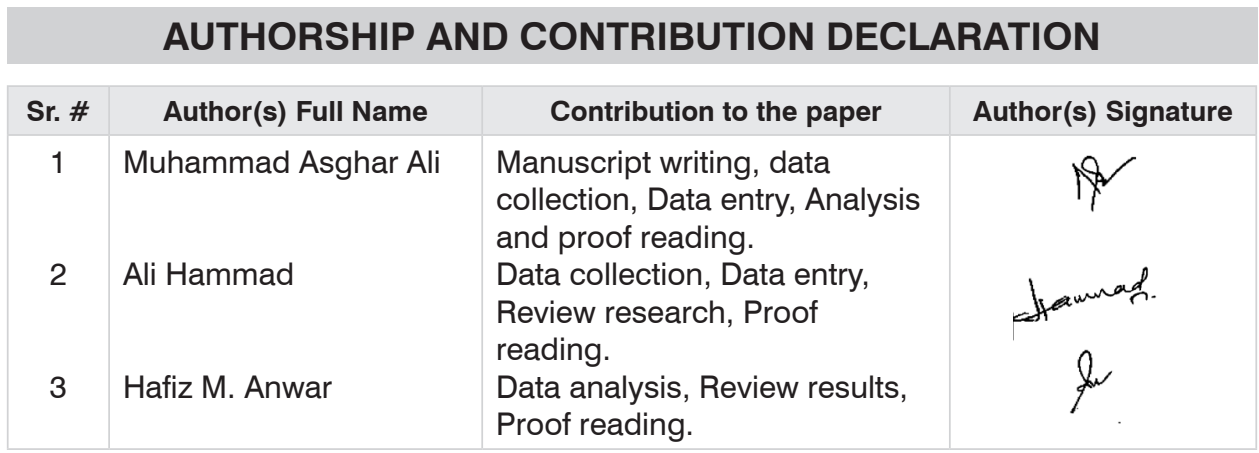

